\title{
La presencia de la arquitectura religiosa latinoamericana en el Premio Internazionale di Architettura Sacra Frate Sole
}

Latin-American Church Architecture in the Premio Internazionale di Architettura Sacra Frate Sole

Giorgio Della Longa · Consulta dell'Ufficio Liturgico Nazionale de la Conferenza Episcopale Italiana (Roma, Italia)

https://doi.org/10.17979/aarc.2015.4.0.5135

\section{RESUMEN}

Este texto pretende analizar la presencia latinoamericana en las cinco ediciones del Premio Internazionale di Architettura Sacra Frate Sole (1996-2012). Una presencia que se ha ido incrementando con el paso de los años hasta lograr su zenit en la última edición con el primer premio concedido a la Capilla en Auco, de Undurraga-Deves.

Conviene recordar el carácter específico del Premio Internacional Frate Sole (en cada convocatoria sólo se pueden presentar las iglesias terminadas diez años atrás), así como su vocación inclusiva, contemporánea y universal de promoción de la arquitectura sacra.

Se analizarán las propuestas significativas —más allá de las premiadas_ recibidas de los países latinoamericanos, para incidir en su especificidad. Los resultados obtenidos ayudan a valorar la importancia de una región católica, como es Latinoamérica, en el discurso arquitectónico mundial.

\section{PALABRAS CLAVE}

Arquitectura religiosa, Latinoamérica, Premio Internazionale Frate Sole, capillas.

\section{ABSTRACT}

This essay is an analysis of the Latin-American participation in the five editions of the Premio Internazionale di Architettura Sacra Frate Sole (International Contest of Church Architecture Frate Sole) 1996-2012. It was a significant and relevant presence, which had been increasing in time and it reached its heyday in the First Prize given to the Cappella of Auco of Undurraga-Deves during the latest edition of the international event.

It's due to mention the particular character of the International Contest -in each edition only the churches built in the previous decade can be submitted - and its peculiar contemporary and universal vocation to promote the Church architecture.

The meaningful proposals, besides the rewarded ones, which came from the Latin-American countries, will be analyzed to contextualize their peculiarities. The results obtained in these years mean to suggest how important the Catholic religion, like the Latin-American one, is in the context of the worldwide Church Architecture.

\section{KEYWORDS}

Sacred Architecture, Latin América, Premio Internazionale Frate Sole, Chapels. 


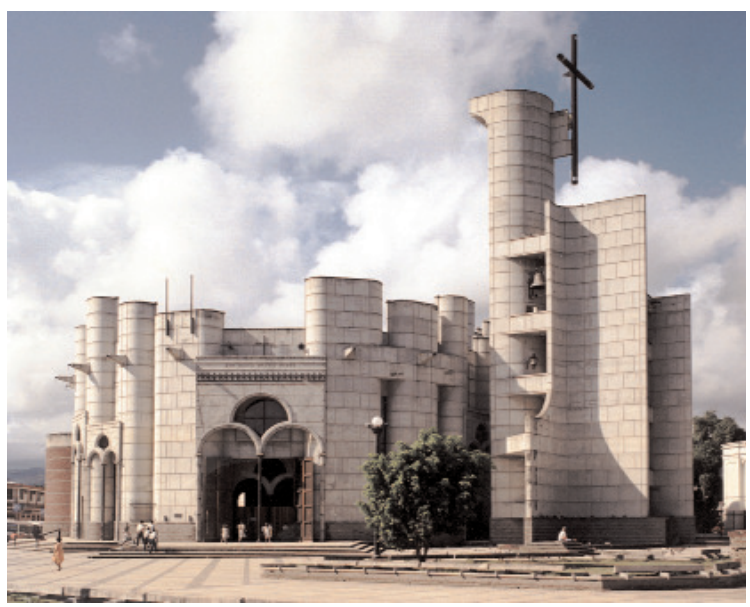

Fig. 01. Pedro Mena Lajara, Catedral de la Inmaculada Concepción, La Vega (República Dominicana), 1977/92.

1

El Premio Internazionale di Architettura Sacra, promovido por la Fondazione Frate Sole, de Pavia (Italia), fue intensamente deseado por el padre Costantino Ruggeri, hermano franciscano y artista, fallecido en el año 2007.

La obra del padre Costantino ha recibido amplio reconocimiento en el ámbito del arte y de la arquitectura sacra contemporánea.

La Fundación tiene entre sus objetivos el de sensibilizar y promover la iglesia construida, para que sea reconocida la componente artística y la tensión mística que elevan el espacio a lugar de exaltación espiritual.

La Fundación ha instituido un premio cuadrienal para reconocer al autor de una obra que haya cualificado significativamente la arquitectura cristiana contemporánea. El premio está dirigido a los nuevos edificios religiosos realizados en todo el mundo en el ámbito de las confesiones cristianas.

Son cinco las ediciones de Premio Internazionale que se han celebrado desde 1996, con una numerosa participación de arquitectos - trescientos setenta, trabajando individualmente o en grupo - provenientes del mundo entero. Los arquitectos latinoamericanos han sido, en total, cuarenta.

Aunque conocido, éste es el listado de los vencedores: Tadao Ando (1996), Álvaro Siza (2000), Richard

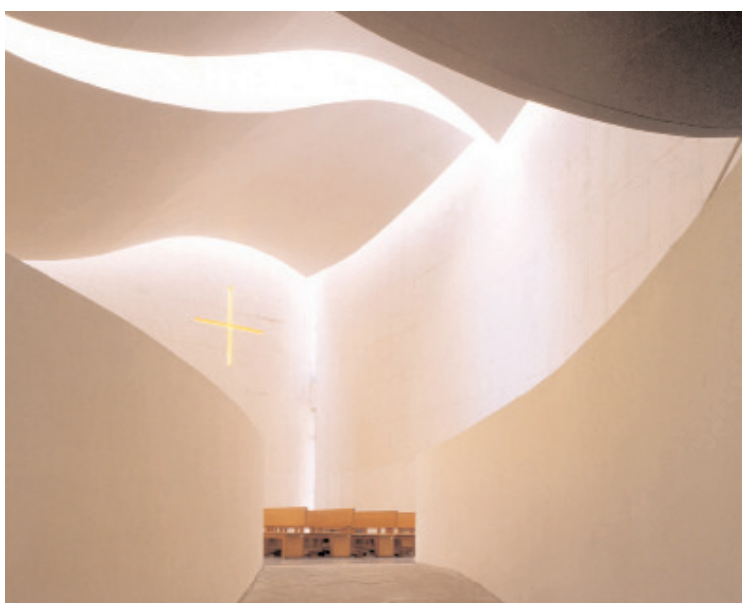

Fig. 02. Enrique Browne y asociados, Capilla del colegio Villa María Academy, Santiago de Chile (Chile), 1992/96.

Meier (2004), John Pawson (2008) y el chileno Cristian Undurraga en la última edición del premio (2012). Toda la documentación relativa a los trabajos enviados a las distintas ediciones del Premio Internazionale está disponible en el sitio de la Fondazione Frate Sole (www.fondazionefratesole.org).

2

Desde hace varios años, además de formar parte del Comité Científico de la Fundación, participo en el Jurado.

Debo hacer una confesión. Creo que supone una seria limitación el que — por razones claramente comprensibles - los que participamos en el jurado, difícilmente hayamos estado en contacto directo con las obras juzgadas.

Yo he podido visitar alguna iglesia, generalmente después de terminar los trabajos de la comisión. Sólo en ese momento he podido comprender el verdadero alcance de la obra, lo que a veces me ha llevado a cambiar notablemente la opinión que tuve como jurado.

Estoy convencido de que sólo el contacto real con la obra permite apreciar su significado y medir su calidad. Por el contrario, el riesgo de premiar al mejor reportaje fotográfico de arquitectura siempre es muy grande.

Con esta necesaria premisa voy a analizar la rica contribución ofrecida por América Latina. 
Fig. 03. Manoel Coelho, Templo da Paz, Universidade Positivo, Curitiba (Brasil), 2002.

Fig. 04. Daniel Bonilla arquitectos, Capilla del colegio Los Nogales, Bogotá (Colombia), 2000/01.

Fig. 05. Daniel Bonilla arquitectos, Capilla Porciúncula de la Milagrosa, La Calera, Bogotá (Colombia), 2003/04. Fig. 06-07. Decio Tozzi, Capilla de la Fazenda Veneza, Valinhos (Sao Paulo, Brasil), 2002.
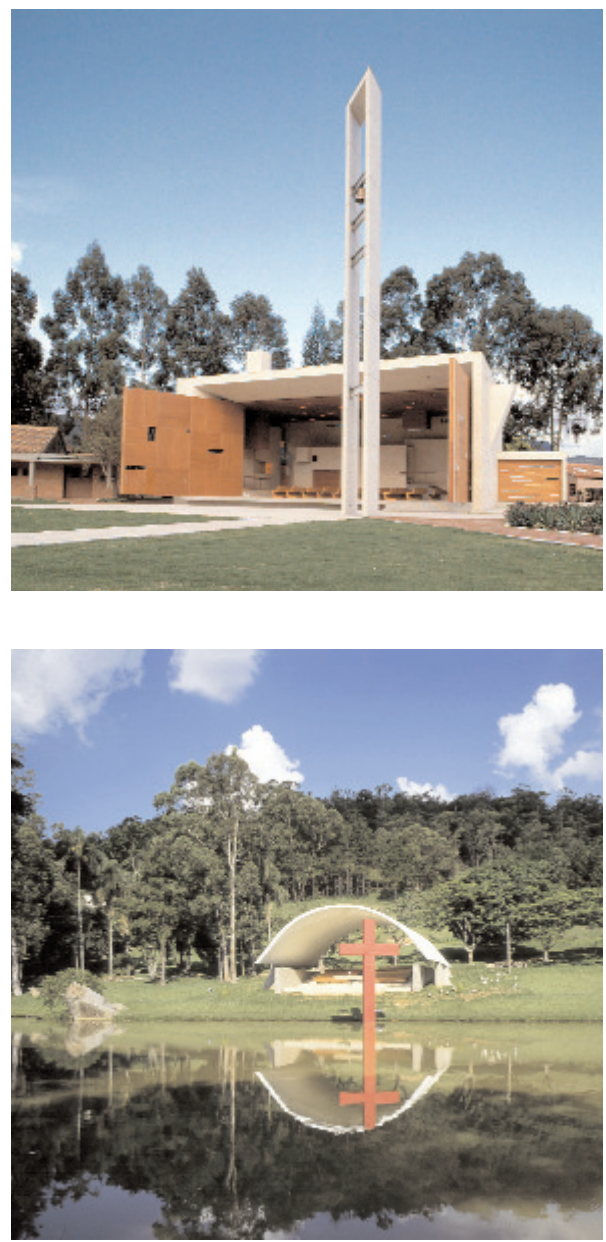
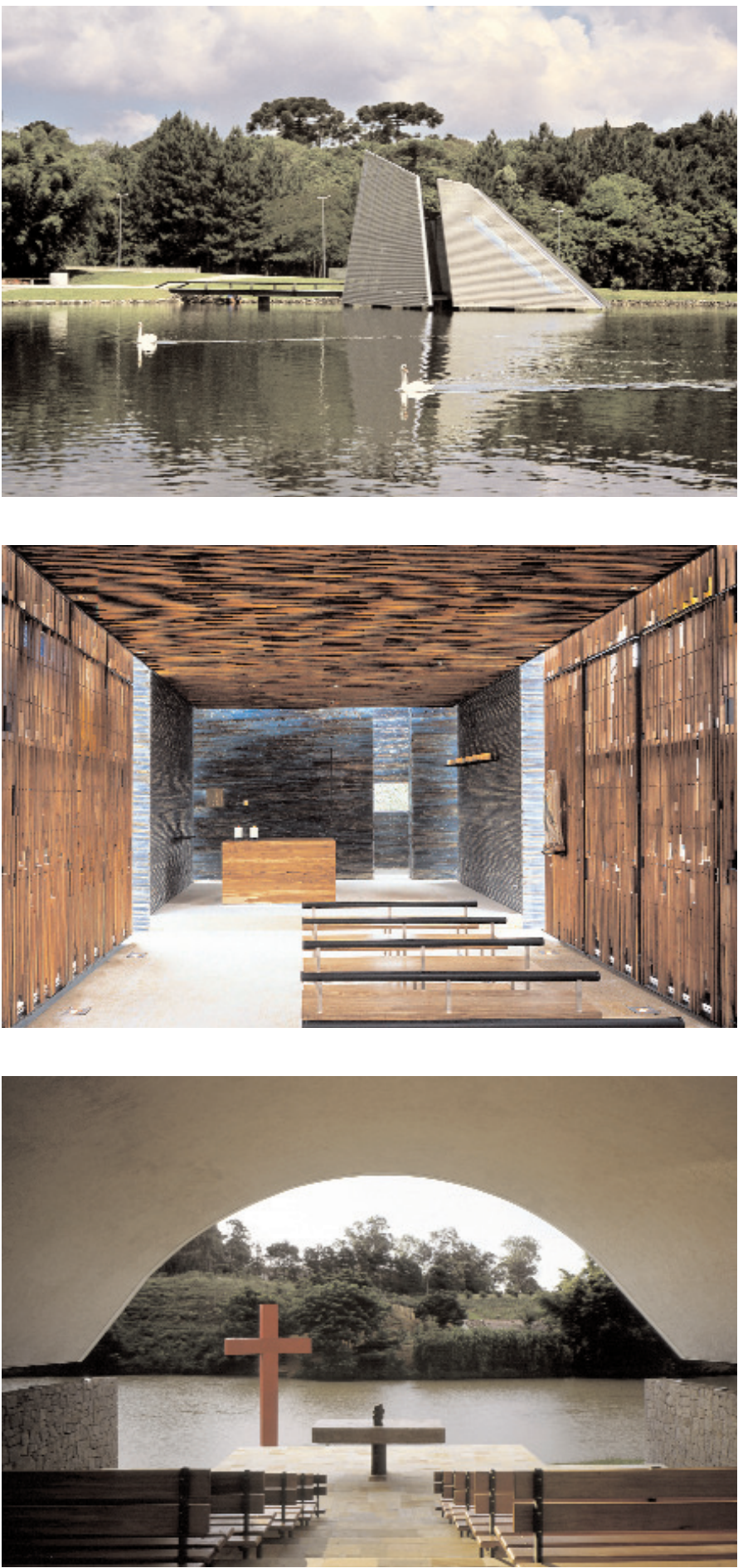

Actas del Congreso Internacional de Arquitectura Religiosa Contemporánea 4 (2015) 
3

En la primera edición del premio (1996), los participantes latinoamericanos no parecieron haber comprendido del todo el objetivo del evento. De Ecuador y Bolivia llegaron testimonios de imponentes basílicas enrocadas en la tradición.

La catedral de la Inmaculada Concepción, en La Vega (República Dominicana), es distinta, por la búsqueda de un equilibrio entre la monumentalidad y el lenguaje moderno (Fig. 01).

\section{4}

En la edición del año 2000, el chileno Enrique Browne recibe una mención especial compartida con los alemanes Hoger y Hare y los noruegos Lund y Slaatto por la capilla de un colegio en Santiago de Chile. Se trata de un espacio para el culto, anexo al preexistente colegio Villa María Academy, de la Congregación de las Hermanas Siervas del Inmaculado Corazón de María (Fig. 02). El edificio se distingue por el imaginativo lenguaje que, en este caso, interpreta el efecto de claroscuro de un cielo en el que se captura el movimiento de las nubes. Los rasgones de luz generan este tipo de sugestión. Parece un espacio dibujado por el soplo del viento, metáfora del Espíritu Santo.

Este edificio anticipa lo que será el rasgo mas característico de las propuestas procedentes de los países latinoamericanos: la pequeña dimensión. Aunque aquí se expresa en términos alegóricos, existe la tensión hacia el espacio abierto, en este caso, la inmensidad del cielo. Y sobre los edificios que tienen esta característica se focaliza este texto.

\section{5}

En la edición de 2004, entre las propuestas presentadas destacan, precisamente, las intervenciones de pequeña dimensión: el Templo de la Paz, ubicado en el interior de la Universidade Positivo, en Curitiba (Brasil), de Manoel Coelho (Fig. 03), y la capilla de la Fazenda Veneza, en Valinhos, Sao Paulo, de Decio Tozzi (Fig. 06-07), ambos arquitectos brasileños.

En esta edición, el jurado señaló dos obras del colombiano Daniel Bonilla, por la «fuerte calidad poética del espacio, obtenida con el sabio uso de los fenómenos lumínicos, el empleo de los materiales y la pureza de la composición» (Fondazione 2004, 38). Se trata de las capillas del colegio Los Nogales y de la
Porciúncula de la Milagrosa, en las cercanías de Bogotá (Colombia), edificios promovidos por comitentes privados (Fig. 04-05). Dos intervenciones muy publicadas en Europa, caracterizadas por una dinámica relación entre interior y exterior.

La primera capilla es un prisma elemental al servicio del culto en el colegio Los Nogales, institución educativa fundada para promover la formación católica. Un espacio interior para un centenar de fieles que puede abrir sus puertas a una comunidad de dos mil personas reunidas al aire libre. Estas grandes puertas son el rasgo más característico de la capilla, un gesto no sólo simbólico, sino funcionalmente acogedor.

La capilla de la Porciúncula de la Milagrosa es un espacio minimalista, caracterizado por una geometría esencial determinada por un precario diafragma que pone el interior en diálogo con el exterior. De hecho, la cambiante relación con la naturaleza — con el silencio, el viento y la luz - constituye la esencia del proyecto.

En ambas capillas, el cambio de orientación entre la axialidad del espacio exterior y la del interior, genera una cruz virtual de uso.

\section{6}

En el año 2008, a pesar de la escasa participación latinoamericana, recibió una mención un proyecto latinoamericano. Es la capilla de la Fazenda Veneza, que su autor, el brasileño Decio Tozzi, volvió a presentar tras su participación en 2004 (Fig. 06-07).

Encargada por un comitente privado, la pequeña capilla al aire libre está conformada por dos elementos plásticos que determinan un espacio en continuidad con el paisaje: una cubierta curva, que protege al hombre, precede a una gran cruz que surge del agua. Este poético lugar de culto, privado de muros, entra así en diálogo con la poderosa y cambiante naturaleza.

Sebastián Vega, Fernando Bravo y Francisco Allard fueron los autores de la capilla del Buen Pastor, pequeña obra prefabricada y de bajo coste realizada en La Ligua, a medio camino entre la imagen de una capilla y la de una típica casa rural (Fig. 08).

7

Significativa - tanto en cantidad como en calidad- fue la participación de arquitectos latinoamericanos en la siguiente edición, la última, cronológicamente hablando. 

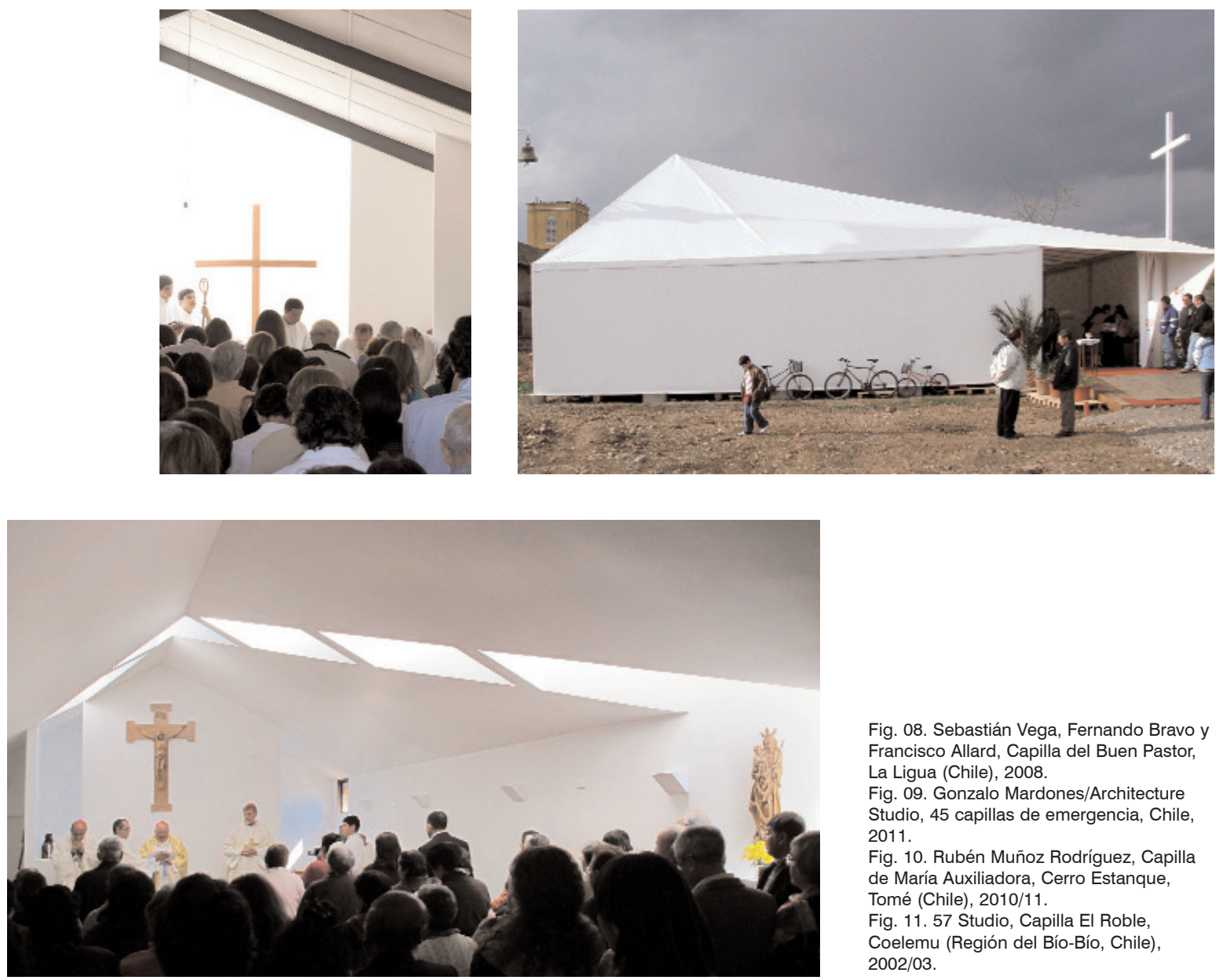

Fig. 08. Sebastián Vega, Fernando Bravo y Francisco Allard, Capilla del Buen Pastor La Ligua (Chile), 2008.

Fig. 09. Gonzalo Mardones/Architecture Studio, 45 capillas de emergencia, Chile, 2011.

Fig. 10. Rubén Muñoz Rodríguez, Capilla de María Auxiliadora, Cerro Estanque Tomé (Chile), 2010/11.

Fig. 11. 57 Studio, Capilla El Roble, Coelemu (Región del Bío-Bío, Chile), 2002/03.

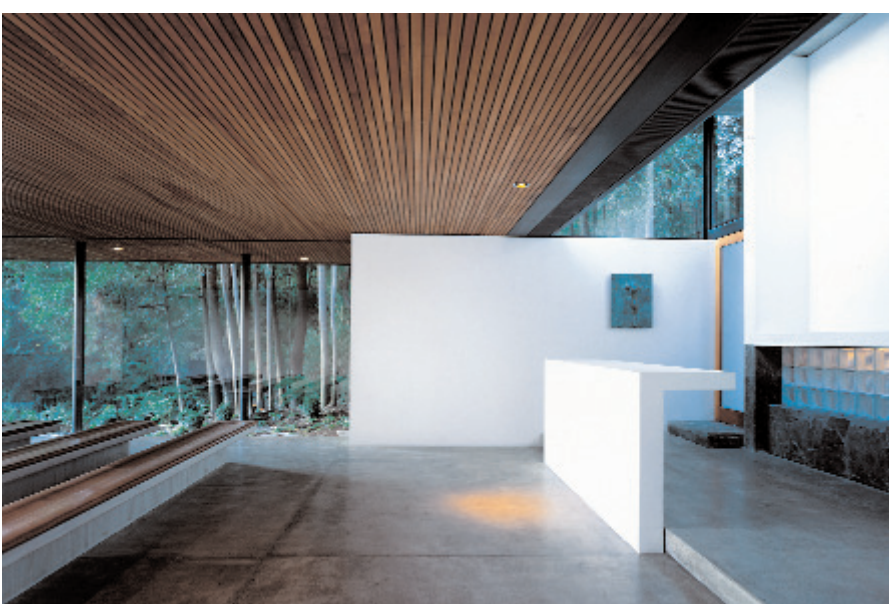


En esta ocasión, las iglesias de pequeñas dimensiones encuentran un propósito de dramática necesidad. Chile es duramente golpeado por el terremoto de 2010 y el ochenta por ciento de los edificios de culto del área afectada quedan fuera de uso. Así, se otorgó una distinción a las pequeñas construcciones promovidas por AIS (Fundación de Ayuda a la Iglesia que Sufre). Lo que se pedía era la realización de un prototipo de capilla para las comunidades rurales de las regiones centrales de Chile que habían sido golpeadas por el terremoto. Las cuarenta y cinco Capillas de emergencia: Reconstruyamos Chile con Cristo son intervenciones de bajo coste y de rápida construcción, que pueden adaptarse a las específicas condiciones climáticas y culturales. La blanca cubierta de la capilla es una metáfora del manto de la Virgen del Carmen, que cubre y protege a los fieles, como expreso recordatorio de la devoción popular (Fig. 09). El autor de las capillas de emergencia es el arquitecto chileno Gonzalo Mardones Viviani.

Análogamente, la archidiócesis de Concepción encarga una capilla para sustituir a una iglesia destruida por el terremoto. La capilla María Auxiliadora, en Cerro Estanque, proyectada por Rubén Muñoz Rodríguez, es una pequeña construcción orientada al este, revestida en acero cortén y madera, con un interior blanco marcado por un rayo de luz (Fig. 10).

Un comitente privado solicita al grupo chileno 57 Studio la capilla El Roble, en la región del Bío-Bío, Chile. Se trata, una vez más, de una capilla de alta calidad arquitectónica, una elegante construcción rodeada de vegetación y caracterizada por la relación con el ambiente natural en el que se inserta. Un gran paño de vidrio vincula el pequeño espacio con el bosque de acacias que lo circunda (Fig. 11).

La edición de 2012 proclama vencedor al chileno Cristian Undurraga, con la Capilla del Retiro en el valle de Los Andes (Chile) (Fig. 12). Encargada por el monasterio carmelita de Auco, la capilla se coloca al lado de la Casa de Espiritualidad del santuario de Santa Teresa de los Andes, situado al pie de los Andes centrales chilenos, lugar de recogimiento y oración para los peregrinos.

La excavación es el gesto primario para enraizar cualquier edificio eclesial, pero en este caso la excavación permanece abierta, y en su interior, un entramado reticular de vigas sostiene el elemento cúbico protector (Fig. 13). El recogido espacio cultual se expande gra- cias a un paño de vidrio que lo prolonga en la naturaleza. Parece que no hubiera solución de continuidad entre el interior y el exterior; éste es el carácter determinante del espacio. Creo que el fiel ha de sentirse fuertemente influido por encontrarse enteramente rodeado por la áspera naturaleza del lugar, y protegido — pero no confinado - por el pseudo-ciborio suspendido.

Concluimos con un grupo procedente de México, el país que nos acoge. Se trata de BNKR Arquitectura, el despacho autor de dos obras límite que sólo convencionalmente podríamos denominar capillas.

La primera, promovida por la empresa Jardín La Estancia, está situada en el interior de un bellísimo jardín en Cuernavaca (México), en el cual se organizan ceremonias nupciales (Fig. 14). Cuernavaca es una ciudad muy popular para las bodas, y este edificio está pensado justamente para celebrar el rito del matrimonio. El espacio está delimitado de modo discontinuo por tabiques acristalados: luz, viento, sonido y perfume penetran en el aséptico interior marcado por una gran cruz en el fondo.

El segundo trabajo parte de un concepto opuesto: una capilla para llorar la muerte de un ser querido. Es la Capilla del Atardecer, también situada en el interior de un magnífico jardín, sobre la bahía de Acapulco (México). La petición del comitente era la de poder gozar desde la capilla de la magnífica vista del paisaje circundante, y además, que estuviese orientada exactamente para recibir el sol en la cruz los días del equinoccio. Poéticamente áspera - un prisma escultórico realizado en hormigón armado pero también abierto a la luz y al aire-, la capilla parece una roca que se mantiene en precario equilibrio entre los bloques graníticos de la cima de la montaña (Fig. 15).

\section{8}

En conclusión, creo que es destacable la significativa contribución de los países latinoamericanos al Premio, dentro del cual sobresale especialmente la atractiva obra proveniente de Chile, cuya participación ha sido coronada en 2012 con el máximo reconocimiento otorgado a Cristian Undurraga.

9

Una observación sobre el espacio abierto.

Las capillas de indios o capillas abiertas mexicanas suelen ser consideradas como la aportación arquitectó- 

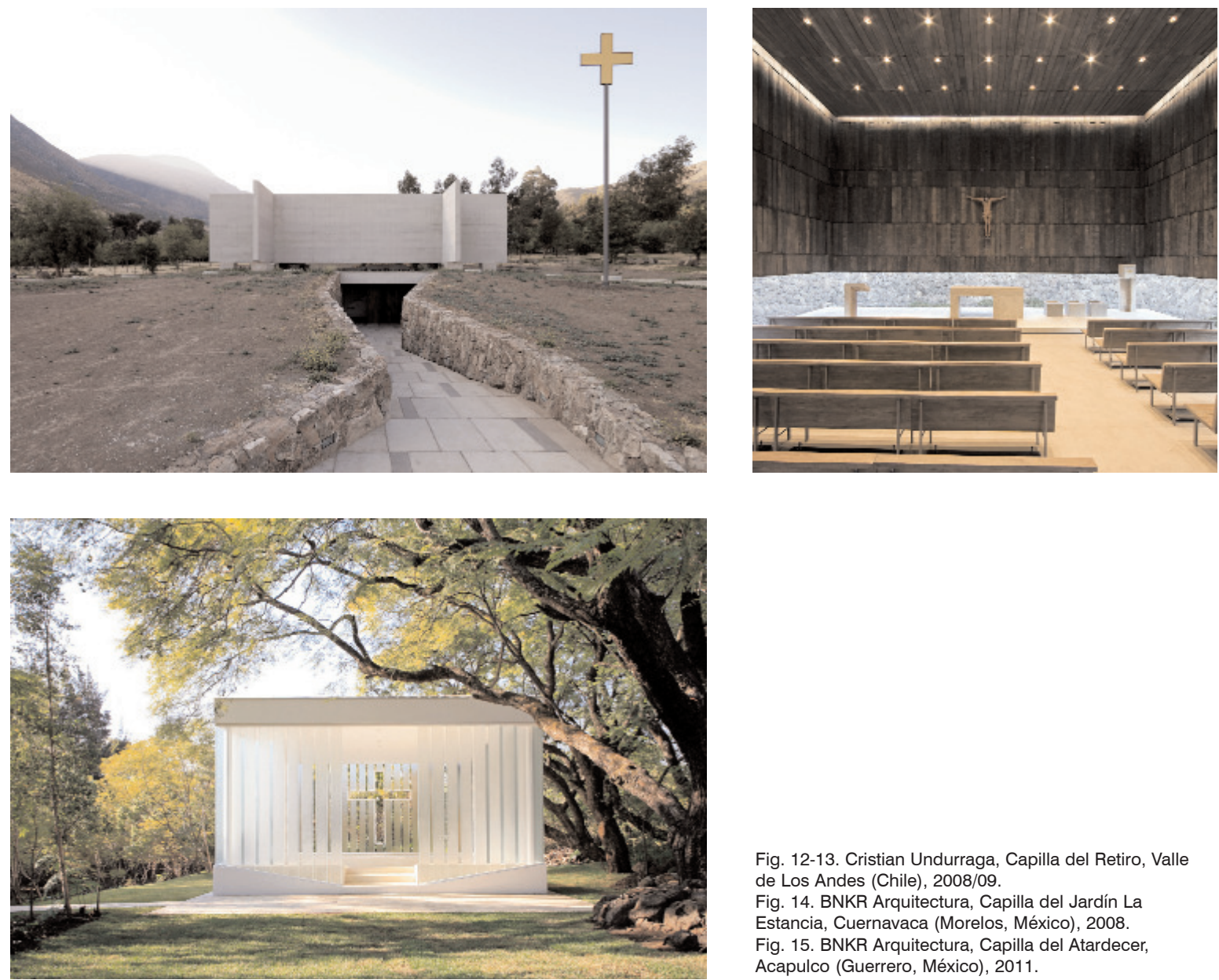

Fig. 12-13. Cristian Undurraga, Capilla del Retiro, Valle de Los Andes (Chile), 2008/09.

Fig. 14. BNKR Arquitectura, Capilla del Jardín La Estancia, Cuernavaca (Morelos, México), 2008. Fig. 15. BNKR Arquitectura, Capilla del Atardecer, Acapulco (Guerrero, México), 2011.

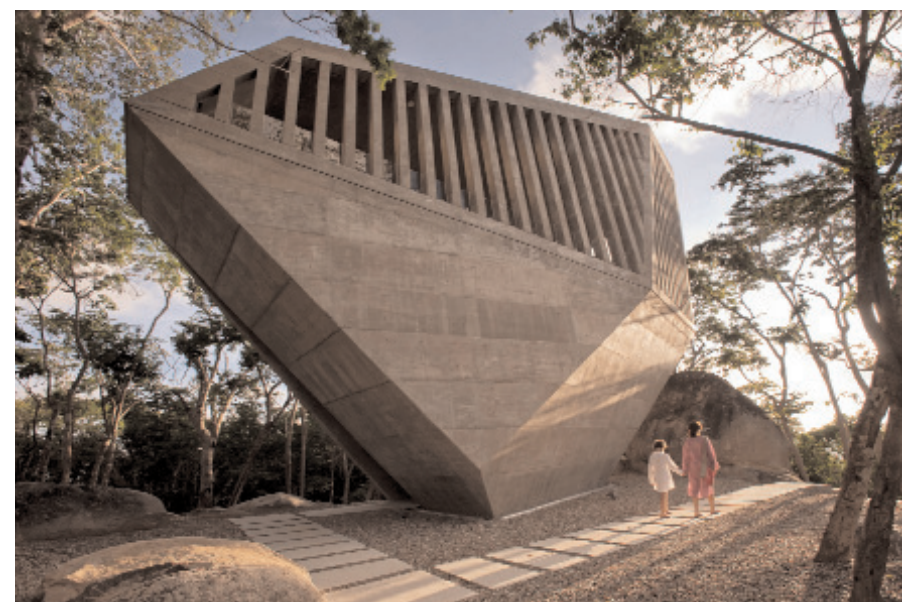


nica más original de la cultura americana hasta la llegada del rascacielos. Los misioneros españoles comenzaron a instruir a los nuevos cristianos bajo construcciones temporales, es decir, simples altares protegidos por un techo de paja o de madera. Una solución que favorecía el amoldamiento de los indígenas a los nuevos ritos, desarrollados en el amplio espacio abierto que para ellos era habitual. Con el paso del tiempo, aquellas capillas abiertas adoptaron formas permanentes, con un carácter propio.

Su herencia ha sido retomada de diversas formas en la arquitectura moderna latinoamericana, desde la capilla que Félix Candela realizó en Lomas de Cuernavaca a la extraordinaria capilla de San Pedro, cerrada sólo con vidrio, de Paulo Mendes da Rocha.

Las pequeñas iglesias presentadas aquí constituyen el testimonio de la incesante puesta al día de esta tradición.

10

Una observación sobre la pequeña dimensión.

Realmente, parece que la expresión de la buena arquitectura religiosa en América Latina se vea favorecida por la pequeña dimensión; es sorprendente el número y la calidad de las obras de este tipo.

Los clientes se han confiado a buenos arquitectos para construir obras a veces cuestionables, pero — ciertamente- de notable interés.

Como observador, me pregunto si también los complejos parroquiales, los edificios de mayores dimensiones y funcionalmente más complejos, que continúan surgiendo en América Latina, cuentan con el apoyo de unos promotores igual de lúcidos y dispuestos a arriesgarse. Me temo que no es así.

He querido, en todo momento, fijarme en el comitente. Si excluimos la institución que patrocinó las capillas de emergencia tras el terremoto de Chile, todos los promotores de las obras son simples particulares $\mathrm{u}$ organizaciones privadas.

Este hecho arroja una luz especial sobre las obras presentadas. Tengo la impresión de que nos encontramos frente a ocasiones afortunadas, a trabajos destinados a una élite. Una élite capaz de promover buena arquitectura, pero que representa muy poco — piensodentro de los complejos fenómenos de religiosidad que existen en esta parte del mundo.

La exigencia de la Iglesia Católica y de sus iglesias — las iglesias de todos los días - parece así escapar al observador externo, y probablemente queda representada mejor por las iglesias recibidas en la primera edición del premio - las grandes basílicas para las multitudes- que por los refinados trabajos que aquí he mostrado.

No puedo menos que recordar ahora el último número de la revista Chiesa e Quartiere, aquél sobre América Latina. Sus artículos - cualquiera de ellosnos arrojan a la cara el dramático problema de la población más pobre, de las periferias marginadas, mientras Europa vivía en aquellos años el llamado boom económico. Giuliano Gresleri, en el prólogo, reclama nuestra atención para la región «più dolorante, più innascoltata, più povera del mundo» (más sufriente, más desatendida, más pobre del mundo) (Gresleri 1968, 2).

Pero a distancia de cincuenta años, a Pavia no parece haber llegado el eco de cómo este dramático estado de la cuestión haya evolucionado, ni de qué respuestas se hayan dado en términos de arquitectura.

\section{BIBLIOGRAFÍA}

«Fondazione Frate Sole», consultado el 1 de octubre de 2015, www.fondazionefratesole.org.

Fondazione Frate Sole, ed. 1996. Premio Internazionale di Architettura Sacra «Frate Sole», I edizione. Tadao Ando. Pavia: Fondazione Frate Sole.

Fondazione Frate Sole, ed. 2000. Premio Internazionale di Architettura Sacra «Frate Sole», II edizione. Álvaro Siza. Pavia: Fondazione Frate Sole.

Fondazione Frate Sole, ed. 2004. Premio Internazionale di Architettura Sacra «Frate Sole», III edizione. Richard Meier. Pavia: Fondazione Frate Sole.

Gresleri, Giuliano, 1968. "Quid agam?», Chiesa e Quartiere 46/47:2-3.

Vaccari, Andrea, ed. 2008. Premio Internazionale di Architettura Sacra «Frate Sole», IV edizione. Milán: Skira.

Vaccari, Andrea, ed. 2012. Premio Internazionale di Architettura Sacra «Frate Sole», V edizione. Milán: Skira.

\section{PROCEDENCIA DE LAS ILUSTRACIONES}

Fig. 01-15. Archivo de la Fondazione Frate Sole (Pavia, Italia).

El autor agradece al arquitecto Andrea Vaccari por el material y la documentación facilitados. 